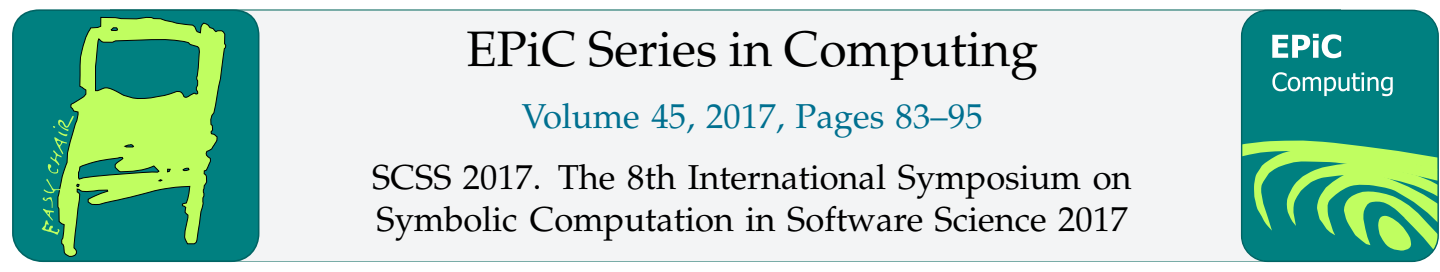

\title{
Origami Folds in Higher-dimension
}

\author{
Tetsuo Ida ${ }^{1}$ and Stephen M. Watt ${ }^{2}$ \\ 1 Faculty of Engineering, Information and Systems \\ University of Tsukuba \\ Tsukuba 305-8573, Japan. \\ ida@cs.tsukuba.ac.jp \\ 2 David R. Cheriton School of Computer Science \\ University of Waterloo \\ Waterloo, Canada N2L 2W1 \\ smwatt@uwaterloo.ca
}

\begin{abstract}
We present a generalization of mathematical origami to higher dimensions. We briefly explain Huzita-Justin's axiomatic treatment of mathematical origami. Then, for concreteness, we apply it to origami on 3-dimensional Euclidean space in which the fold operation consists of selecting a half-plane and reflecting one half-plane across it. We finally revisit the subject from an $n$-dimensional point of view.
\end{abstract}

\section{Introduction}

Origami, the Japanese art of paper folding, allows the creation of a wide range of beautiful figures. Just as drawing with a straightedge and a compass define a class of idealized constructible figures, so do the fold operations of origami. The class of figures that may be so formed strictly includes those that may be formed with a straightedge and a compass. For example, it is possible to construct a regular heptagon with origami folds, while it has been shown impossible by a straightedge and a compass(as a consequence of [17]). The mathematical properties of origami have been well-studied from several points of view, including plane geometry, axiomatic systems and software implementation.

The basic operation of origami is the fold. From a mathematical point of view, a fold operation may be defined by a reflection along an oriented line $\ell$ in the plane. Suppose that we employ the right-handed system. The half-plane to the right of $\ell$ is reflected onto the the halfplane to the left. The fold operation is viewed as a mapping from point sets to point sets need not refer to any notion of an embedding space in which the fold takes place. This enables us to reason origami more abstractly, and leads us to study origami in higher-dimensional settings.

We consider a generalization of mathematical origami to higher-dimensions. We first formalize Huzita-Justin's pioneering works[9][14] on mathematical origami. Then for concreteness, we begin our exploration with origami on 3-dimensional Euclidean space in which the fold operation consists of selecting a half-plane and reflecting one half-plane across it. We then revisit the subject from an $n$-dimensional point of view. 


\section{Axiomatization of 2-dimensional Origami Geometry}

\subsection{Huzita-Justin Fold Operations}

Huzita [9] and Justin [14] gave the set of elementary fold operations, independently in the same proceedings ${ }^{1}$. It is based on the following two observations on the paper folds. (i) for arbitrarily given distinct points on a sheet of paper, i.e., an origami, we can construct, by hand, a line segment, to be called fold-line, that passes through them. (ii) we can superpose, by hand, two distinct points on the origami by a single fold.

Each elementary fold operation in the set takes a form of a command-like expression, logically equivalent to the geometric tool with its well-defined functionality. We call this set $H O$, hereafter. When we compare the geometric construction power of $\mathrm{HO}$ with a straightedge and a compass of Euclidean geometry in Subsection2.2, we will treat $\mathrm{HO}$ a tool of geometric construction, as well.

HO has played a fundamental role in the formalization of the origami geometry $[9,7,3]$, just as the tools of a straightedge and a compass played in the construction of geometric objects in classical Euclidean geometry. In the 2-dimensional (2D) Euclidean geometry, straightedges and compasses are used, whereas HO relies only on fold operations of an origami by hand.

We will first extend $\mathrm{HO}$ to enable us to construct a class of 3D origamis. We focus on the situations that all the points and lines that serve as the parameters to the $\mathrm{HO}$ folds lie on the same plane $\mathcal{P}$, and that the set $\mathcal{F}$ of the origami faces that we fold should lie on that plane. We call $\mathcal{P}$ and $\mathcal{F}$ base plane and base face set of the folds, respectively.

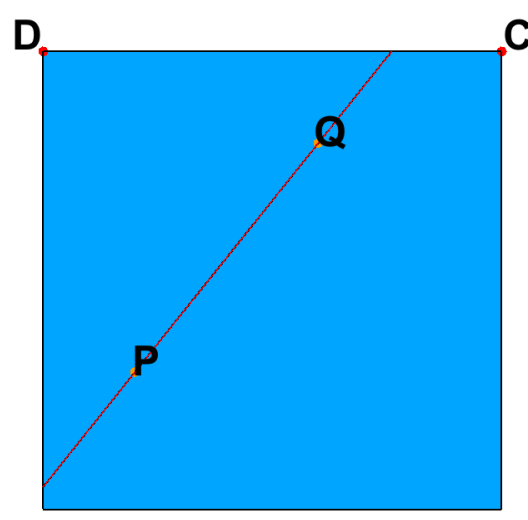

(a) Points $P$ and $Q$ on the origami; red line is a part of the fold line

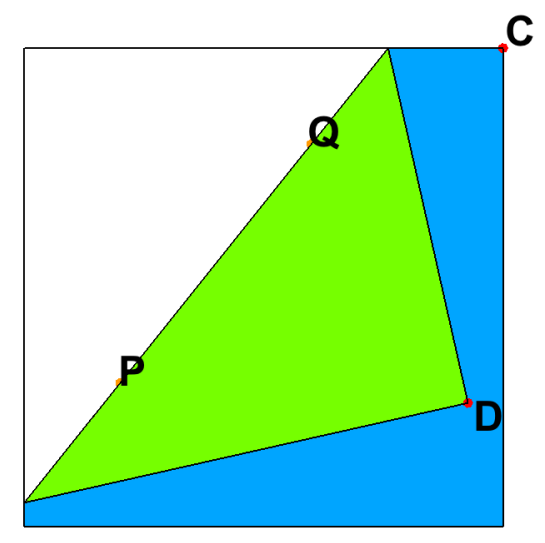

(b) Origami folded along fold-line $P Q$

Figure 1: Fold along the line passing through two points

While constructing, by hand, a fold-line that passes through two distinct points is obvious (see Fig. 1), the superposition (see Figs. 2 and 3) would need some explanation, before we proceed. We consider the superposition of only lines and points. Let us use $\alpha$ and $\beta$ to denote a point, line or a half-line, generically. To express "superpose $\alpha$ onto (or to) $\beta$ and vice versa", we will also say "superpose $\alpha$ and $\beta^{\prime \prime}$.

\footnotetext{
${ }^{1}$ The same paper by Justin appeared also in the local journal of APMEP of Alsace and the IREM of Strasbourg, 1936. In the paper, the author acknowledged the communication with Huzita.
} 


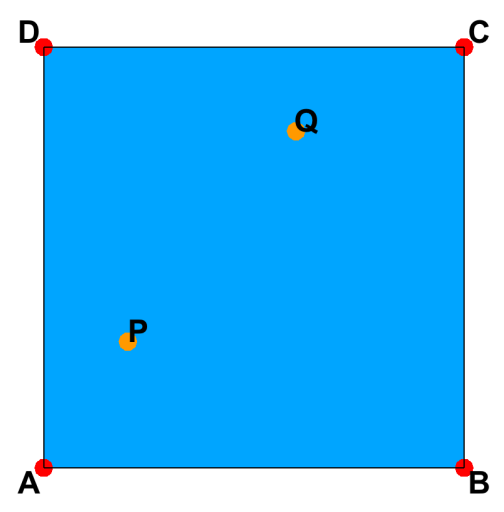

(a) Points $P$ and $Q$ on the origami

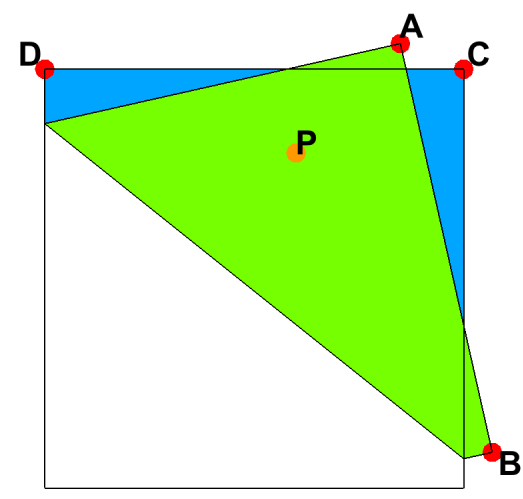

(b) Origami folded to suprpose $P$ and $Q$

Figure 2: Fold to superpose two points

Depending on the configuration of the points and lines under consideration, we distinguish the following six cases of the superposition.

(1) To superpose a point $P$ and a point $Q$ : We assume that it is feasible by hand (cf. Fig. 2(a)(b)).

(2) To superpose a point $P$ onto a line $m$ and vice versa: We assume that it is feasible by hand since we can take an arbitrary point on $m$, and then apply Case (1).

(3) To superpose a half-line $m$ and a half-line $n$, where the two half-lines are concurrent at a point, say $X$. We assume that it is feasible by hand since we can take an arbitrary point (other than $X$ ) on $m$ and superpose it on $n$ with the $X$ fixed. The fold-line is the bisector of the angle at $X$.

(4) To superpose a line $m$ onto a line $n$ : We distinguish further the following cases:

(i) To superpose two distinct lines $m$ and $n$ :

(a) $m$ and $n$ are parallel: We can superpose them by folding along the line that consists of the points equidistant from $m$ and $n$.

(b) $m$ and $n$ are concurrent: There is a unique point, say $X$, incident on the two lines. The point $X$ splits each of the two lines into two half-lines. We take a pair of half-lines $m_{1}$ and $n_{1}$, each taken from $m$ and $n$, respectively, as shown in Fig. 3(a). We can superpose the half-lines $m_{1}$ and $n_{1}$ by applying case (3). The fold-line $\ell$ is the bisector of the angle at $X$. It is one of the fold-lines. The line $\ell$ is also the bisector of the angle at $X$ formed by the half-line pair $\left(m_{2}, n_{2}\right)$. When we take the other half-line pair $\left(m_{2}, n_{1}\right)$, we get the other fold-line that is perpendicular to $\ell$.

When the point $X$ is outside the origami, imagine to enlarge the origami to the extent that it contains $X$. After the superposition, we clip the enlarged origami to its original size. 
(ii) When we superpose a line $m$ onto itself, we split the line $m$, at some point, say $X$, on $m$, into two half-lines $\left(m_{1}, m_{2}\right)$ as shown in Fig. 3(b). The fold-line is the orthogonal to line $m$ and is passing through $X$.

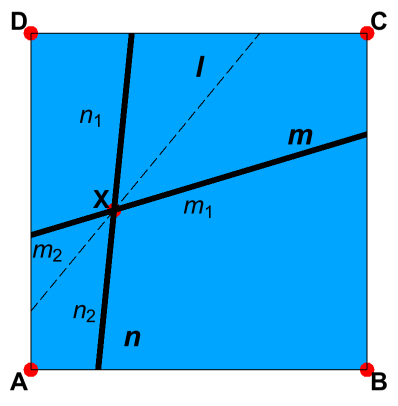

(a) Superposition of concurrent lines

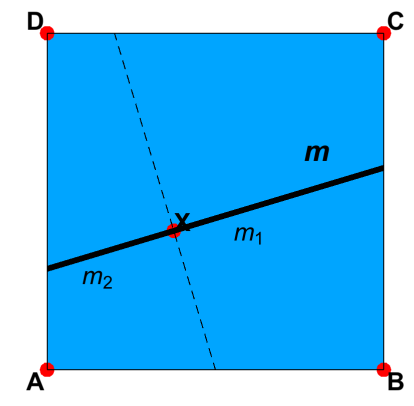

(b) Superposition of a line

Figure 3: Superposition of two lines, and superposition of one line

\subsection{HO: Set of Huzita-Justin Elementary Folds}

We are now ready to present $\mathrm{HO}$, formally. $\mathrm{HO}$ consists of seven statements $(\mathrm{O} 1) \sim(\mathrm{O} 7)$ about the fold operation on the abstract origami. Let $\mathcal{O}=(\mathcal{F}, \succ, \backsim)$ denote an abstract origami [12]. We assume that it is placed on the base plane $\mathcal{P}$. The abstract origami $\mathcal{O}$ is equipped with the set of faces $\mathcal{F}$ with binary relations $\succ$ and $\backsim$ on it, each called superposition and adjacent relations, respectively. It suffices that we remain at this level of abstraction to continue our discussion. Namely, we do not need more abstract or more concrete description about the structures of the origamis in this paper.

We fold an origami $\mathcal{O}$ along a fold-line determined by the parameters of each elementary operation. The parameters are lines each specified by a pair of distinct points on the faces, and points on the faces.

(O1) Given two distinct points $P$ and $Q$, both on $\mathcal{F}$, fold $\mathcal{O}$ along the fold-line on $\mathcal{P}$ that passes through $P$ and $Q$.

(O2) Given two distinct points $P$ and $Q$, both on $\mathcal{F}$, fold $\mathcal{O}$ along the fold-line on $\mathcal{P}$ to superpose $P$ on $Q$.

(O3) Given two distinct lines $m$ and $n$, both on $\mathcal{F}$, fold $\mathcal{O}$ along a fold-line on $\mathcal{P}$ to superpose $m$ on $n$.

(O4) Given a line $m$ and a point $P$, both on $\mathcal{F}$, fold $\mathcal{O}$ along the fold-line on $\mathcal{P}$ passing through $P$ to superpose $m$ onto itself. 
(O5) Given a line $m$, a point $P$ not on $m$, and a point $Q$, where $m, P$ and $Q$ are on $\mathcal{F}$, fold $\mathcal{O}$ along a fold-line on $\mathcal{P}$ passing through $Q$ to superpose $P$ and $m$.

(O6) Given two lines $m$ and $n$, two point $P$ and $Q$ each not on $m$ and not on $n$, respectively, where $m$ and $n$ are distinct or $P$ and $Q$ are distinct, and furthermore $m, n, P$ and $Q$ are on $\mathcal{F}$, fold $\mathcal{O}$ along a fold-line on $\mathcal{P}$ to superpose $P$ and $m$, and $Q$ and $n$.

(O7) Given a line $m$, a point $P$ not on $m$, and a line $n$ distinct from $m$, where $m, n$ and $P$ are all on $\mathcal{F}$, fold $\mathcal{O}$ along the fold-line to superpose $P$ and $m$, and $n$ onto itself.

From $\mathrm{HO}$, we will be able to define an origami geometry in a similar way laid down in the classical Euclid geometry. In Huzita [9], he made an initial attempt to axiomatize an origami geometry in Euclidean way. We will follow the path of his research.

We take the following statements (A1) and (A2) as the axioms of the origami geometry.

Axiom 2.1 (A1). Given two distinct points on $\mathcal{F}$, we can fold $\mathcal{O}$ along the unique fold-line that passes through both points. (cf. Fig. 1.)

Axiom 2.2 (A2). Given two distinct geometric objects $\alpha$ and $\beta$ on $\mathcal{F}$, we can fold $\mathcal{O}$ to superposes $\alpha$ and $\beta$. (cf. Fig. 2, when $\alpha$ and $\beta$ are points.)

Depending on the configurations of the point(s) and the line(s) that are the parameters of each operation $(\mathrm{O} 3) \sim(\mathrm{O} 7)$, its operability, i.e., foldability, is determined, and can be given as propositions. The number of possible fold-lines of each operation is easily determined by algebraic analysis [8]. The well-known results are summarized in Table 1. Note that 0 in the column "number of fold-lines" implies that it is impossible to perform the specified fold.

The powers of $\mathrm{HO}$ used in the origami geometry and the power of the tool of a straightedge and a compass used in the classical Euclidean geometry have been extensibly studied in the past. Wantzel showed that trisecting an arbitrary angle is impossible by a straightedge and a compass [17]. This is due to the inability of finding a cubic root of degree 3 polynomial equations by a straightedge and a compass. A decade later Piazzola Beloch [4] showed that the cubic root of 2 is constructible by the elementary fold operation, which we called (O6) of HO. Martin's work[16], more than 60 years later than Beloch's solidified the significance of (O6). We compare the powers of the two pairs of the tools by the following way. We inductively define the set of constructible points that the both tools can generate. It is easy to convert those points to constructible numbers. The algebraic properties of the constructible number sets are easily characterized. The set of constructible points by $\mathrm{HO}$ is a proper superset of the set of constructible points by a straightedge and a compass [10,2]. See, also, Cox [5](Theorem 10.3.4, page 276) for concise summary on this subject).

\begin{tabular}{|c|l|}
\hline fold operation & number of fold-lines \\
\hline \hline (O3) & 1,2 \\
\hline$(\mathrm{O} 4)$ & 1 \\
\hline$(\mathrm{O} 5)$ & $0,1,2$ \\
\hline (O6) & $0,1,2,3$ \\
\hline (O7) & 0,1 \\
\hline
\end{tabular}

Table 1: Number of possible fold-lines in $\mathrm{HO}$ 


\subsection{Example: Trisecting an Angle}

We show how $\mathrm{HO}$ is used to construct the trisectors of an arbitrarily given angle. Let $E$ be an arbitrary point on the edge $D C$ (see Fig. 4(a)). We construct two lines that trisect $\angle E A B$. This construction is a variant (slightly more general) of Abe's method [1]. We put an arbitrary point $F$ on the edge $D A$ (see Fig. 4(a)). We name this step as Step 1. Then we apply the following elementary operations in sequence. Note that we omit the unfold operation after each execution of Steps 2 and 3. The shown figures are the ones obtained after unfolding. The dashed lines are so-called creases made after the valley-folds.

Step 2: (O4) with $m=A D$ and $P=F$ (see Fig. 4(a))

Step 3: (O2) with $P=A$ and $Q=F$ (see Fig. 4(b))

Step 4: (O6) with $P=F, Q=A, m=A E$ and $n=H I$ (see Fig. 5(a))

There are three possible fold-lines, each trisecting $\pi-\angle E A B, 2 \pi-\angle E A B$ and $\angle E A B$, We choose the third one. Recall that in Table 1, we have three possible fold-lines in the row (O6).

Step 5: (O1) with $P=A 2$ and $Q=A$ (see Fig. 5(b))

Step 6: (O1) with $P=A 2$ and $Q=H$ (see Fig. 6(a))

After Step 6, we unfold the origami twice and obtain the the crease pattern with marked points as shown in Fig. 6(b). For clarity, the two trisectors are drawn in bold and red.

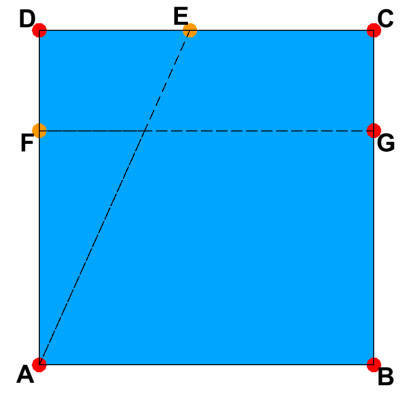

(a) Origami after Step 2

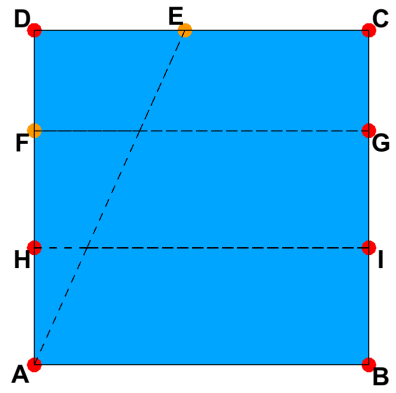

(b) Origami after Step 3

Figure 4: Trisection of $\angle E A B$ (Steps 2 and 3)

The construction shown above was performed by computational origami system Eos[13]. The automated proof for arbitrary point $E$, and point $F$ on line $A D$ is performed by Eos. The prover in this case resorts to Gröbner basis computation of the set of the polynomials whose coefficient domain is rational functions. The set of polynomials is generated automatically from the construction. 

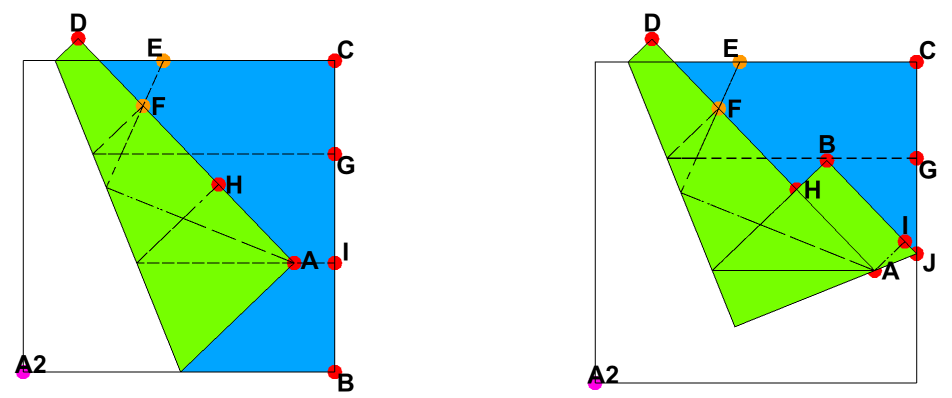

(a) Origami after Step 4

(b) Origami after Step 5

Figure 5: Trisection of $\angle E A B$ (Steps 4 and 5)
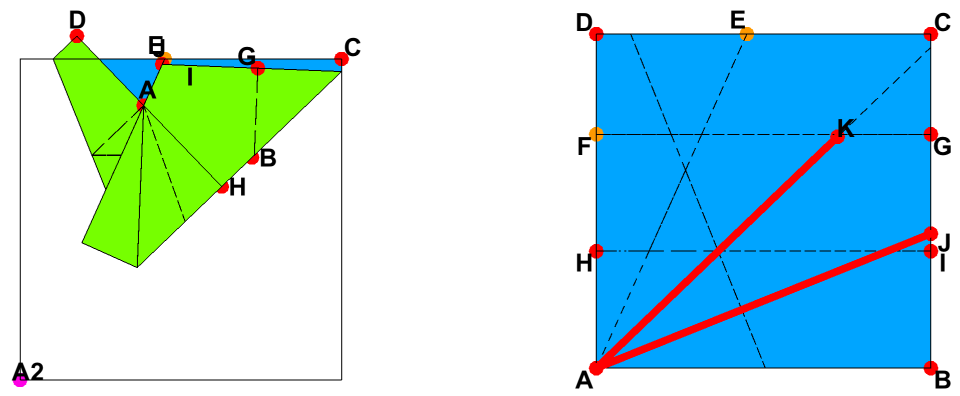

(a) Origami after Step 6

(b) Origami after Step 7

Figure 6: Trisection of $\angle E A B$ (Steps 6 and 7)

\section{Origami of Euclidean 3-dimensional Space}

\subsection{Fold Operations for 3-dimensional Origami}

By analogy of a fold of the plane being a choice of line and reflection of a half-plane, we define a fold of Euclidean 3-dimensional space. In this section, we describe generalizations of HO to 3-dimensional space. In this generalization, a fold-line corresponds either to a fold-line in a plane, i.e., as a $(0+1)$-dimensional object, or as a half-plane, i.e., as a $(3-1)$-dimensional 


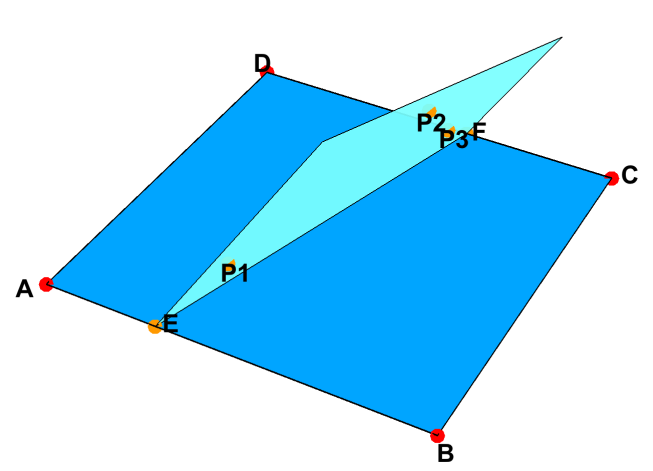

(a) Fold-plane

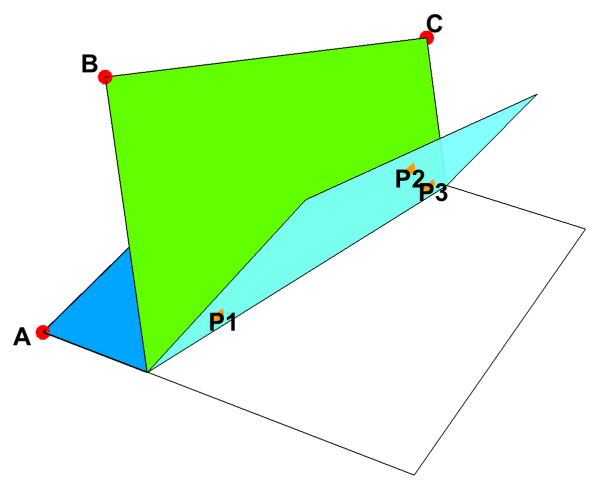

(b) Result of (3D-O1)

Figure 7: (3D-O1) with $P 1, P 2$ and $P 3$

object.

In $2 \mathrm{D}$ origami, the fold consists of two sub-operations:

1. To determine the fold-line. The fold-line divides the origami base plane into two halfplanes, and some faces on the base plane.

2. To rotate one of the half-planes by degree $\pi$ (or $-\pi$ ). The faces on the rotated half-space are also rotated accordingly. The choice of the half-plane and the direction of the rotation remains parametric, i.e., being left to the origami designer.

This rotation realizes the 3D-reflection of faces across the half-plane normal to the base plane and on which the fold-line lies. In 3D origami, we generalize the notion of the fold-line from the rotation by an angle $\pm \pi$ along the fold-line to the rotation about the fold-plane.

In $3 \mathrm{D}$ origami, the reflection is made explicit, rather than hidden behind the fold operations. As a consequence, the fold is the reflection across the fold-plane that is determined by the parameters to the elementary $3 \mathrm{D}$ fold operations. The fold-plane is now a mirror, and the fold is to obtain the image faces in front of the mirror and then to compose them with the faces on the backside of the mirror. To see this more clearly, let us discuss the extension of (O1) to 3D. We will call this elementary fold operation extended to 3D by prefixing "3D-", e.g. (3D-O1).

(3D-O1) Given three distinct points $P_{1}, P_{2}$ and $P_{3}$ on $\mathcal{F}$, that are not collinear, fold $\mathcal{O}$ across a fold-plane on which the three points lie.

When the three points are not collinear, there exists a unique half-plane on which $P_{1}, P_{2}$ and $P_{3}$ lie. Figure 7(a) shows the fold-plane specified by points $P_{1}, P_{2}$ and $P_{3}$. Line $E F$ is the intersection of the origami $A B C D$ and the fold-plane. Figure $7(b)$ shows the combined figure of the result of the (3D-O1) fold and the fold-plane. 


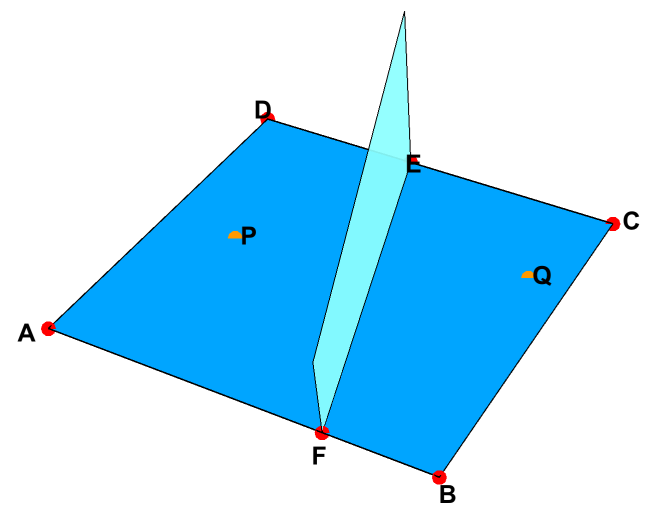

(a) Fold-plane

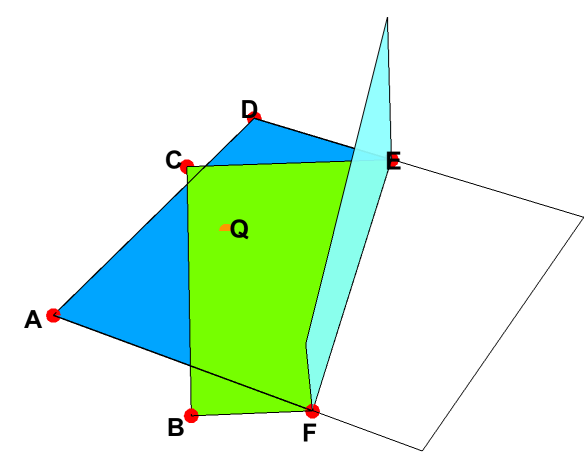

(b) Result of (3D-O2)

Figure 8: (3D-O2) with $P$ and $Q$

(3D-O2) Given two distinct points $P$ and $Q$, fold $\mathcal{O}$ across the fold-plane to superpose $P$ onto Q.

The fold-plane is the plane whose normal (vector) is $\overrightarrow{P Q}$ or $\overrightarrow{Q P}$, and on which the midpoint of $P$ and $Q$ lie (See Figs. 8(a) and 8(b)). Note that the fold planes shown in light blue in Fig. 7(b) and Fig. 8(b) are only for the illustrative purpose. It is not a part of the origami.

(3D-O3) Given two distinct lines $m$ and $n$, that are coplanar, fold $\mathcal{O}$ across a fold-plane to superpose $m$ onto $n$.

The construction of the fold-planes will be discussed in Subsection 3.2 after we introduce (3D-O4), (3D-O5), (3D-O6) and (3D-O7), since we use the same technique of the construction that resorts to the method of the 2D-origami construction.

(3D-O4) Given a line $m$ and a point $P$, fold $\mathcal{O}$ across the fold-plane passing through $P$ to superpose $m$ onto itself.

There is a unique half-plane which is orthogonal to line $m$ and on which point $P$ lies (Figs. 9(a)(b)). This half-plane is the desired fold-plane.

(3D-O5) Given a line $m$, a point $P$ not on $m$, and a point $Q$, where $m, P$ and $Q$ are on the same plane, fold $\mathcal{O}$ along a fold-plane passing through $Q$ to superpose $P$ and $m$.

(3D-O6) Given two lines $m$ and $n$, two point $P$ and $Q$ each not on $m$ and not on $n$, respectively, where $m$ and $n$ are distinct or $P$ and $Q$ are distinct, and furthermore $m, n, P$ and $Q$ are on the same plane, fold $\mathcal{O}$ across a fold-plane to superpose $P$ and $m$, and $Q$ and $n$.

(3D-O7) Given a line $m$, a point $P$ not on $m$, and a line $n$ distinct from $m$, where $m, n$ and $P$ are all on the same plane, fold $\mathcal{O}$ across the fold-plane to superpose $P$ and $m$, and $n$ onto itself. 
Using these operations, it is possible to construct complex figures such as regular polytopes [6], perform dihedral angle divisions, and so on.

\subsection{Extension of 3-dimensional Elementary Fold Operations}

The construction of the fold-plane for the cases of (3D-O3), (3D-O5), (3D-O6) and (3D-O7) are similar to the one for (3D-O4). Let $i$ denote an index of 3, 5, 6 or 7 . For each $i$, we construct the fold-plane as follows. We define the plane, say $\mathcal{W}$, where all the geometric parameters to (3D$\mathrm{O} i$ ) lie. We consider that $\mathcal{W}$ is the base plane of $2 \mathrm{D}$ origami. Then we apply (Oi) to (3D-Oi), and obtain the fold-line. We take a plane that passes through the fold line and is orthogonal to plane $\mathcal{W}$. We split this plane by $\mathcal{W}$ into upper and lower half-planes. The fold-plane is unique for each fold line. Finally, we take one of the half-plane, depending on how we fold the origami (mountain or valley). This half-plane is the desired fold-plane. By construction, the numbers of fold-planes are the same as those for $(\mathrm{O} i)$ given in Table 1.

There are interesting situations where two distinct line parameters are involved in (3DO3), (3D-O6) and (3D-O7). In these cases, obviously, we cannot always assume that the two lines are coplanar. Even in the case that two lines are skew, we can make the 3D fold possible, provided that we allow pre-processing of a rotation of one of the lines such that the rotated line and the non-rotated line lie on the same plane and that the point type parameters become to lie on the same plane. We will discuss this extension for (3D-O3). In (3D-O3), we have no point parameter and the treatment is easier than the other cases. The algorithm is as follows. See Fig. 10 for the referred graphic objects:

1. Take an arbitrary point $X$ that lies on $m$ and does not lie on $n$. See Fig. 9(a).

2. Point $X$ and $n$ form a plane $\mathcal{Q}$ (shaded in green in the sub-figures). Define a location vector $\vec{V}$ that is normal to $\mathcal{Q}$ and whose footing is $X$. Vector $\vec{V}$ and $m$ form a plane $\mathcal{R}$ (shaded in red in the sub-figures). See Fig. 9(b).

3. Rotate $m$ along $\vec{V}$ on $\mathcal{R}$ anchored at $X$ by an appropriate degree $\theta$ such that the moved $m$ and $n$ be coplanar (i.e., $m$ ' will be moved to be on $\mathcal{R}$ ).

4. Let $m$ ' denote the rotated line $m$. Apply (O3) with line parameters $m$ ' and $n$.

\section{The $n$-dimensional Fold}

We used the word fold-line for 2D origami and fold-plane for 3D origami. To extend this usage to higher dimensions, we need to introduce yet another more general terminology for higher dimension origami. We define a fold-hyperplane in $n$-dimensional origami to be a $n$ 1)-dimensional hyperplane. The fold operation reflects one of the half-plane onto the other half-plane across the fold-hyperplane.

( $n$ D-O1) Given $n$ points in general position in $n$-dimensional space, fold $\mathcal{O}$ across the foldhyperplane of $(n-1)$-dimension that passes through all of them.

( $n$ D-O2) Given two distinct points $P$ and $Q$, fold $\mathcal{O}$ across a unique fold-hyperplane that superposes $P$ onto $Q$.

Operation $(n \mathrm{D}-\mathrm{O} 2)$ remains true as stated. The construction is as follows: Let $\ell$ be the fold-line that passes through $P$ and $Q$. Construct the $(n-1)$-dimensional hyperplane orthogonal to $\ell$ and passing through the midpoint of $P$ and $Q$. 


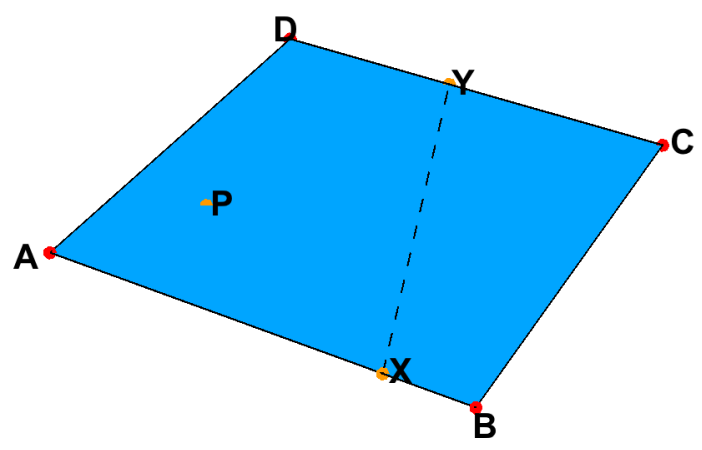

(a) Plane with coplanar parameters in (3D-O4)

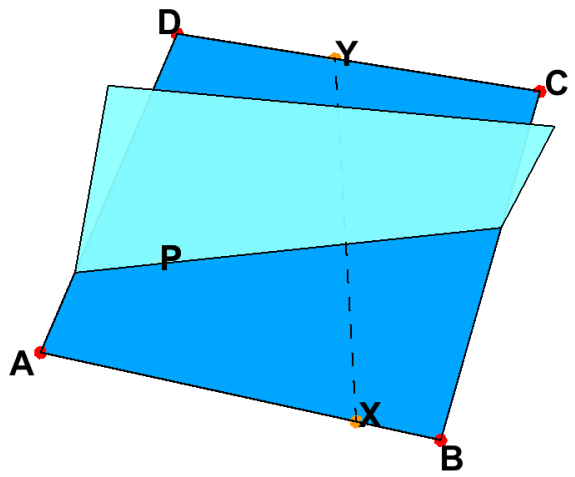

(b) Fold-plane of (3D-O4)

Figure 9: Fold-plane in (3D-O4)

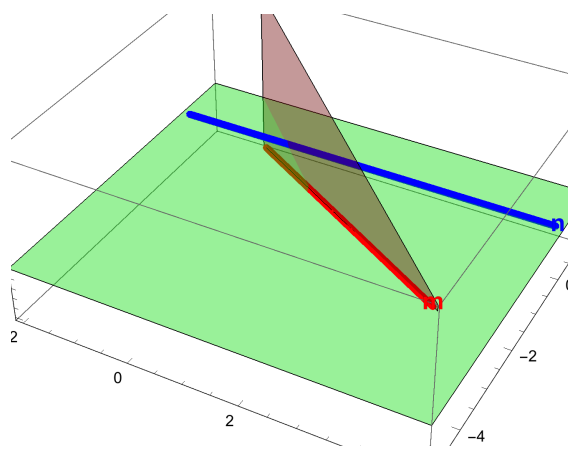

(a) Fold-plane with skew line parameters

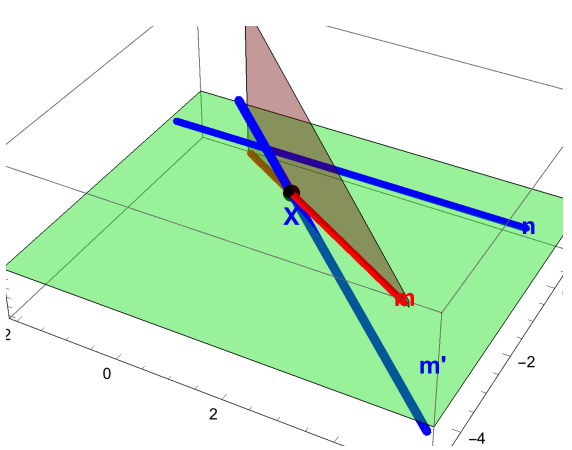

(b) Coplanar line parameters $m^{\prime}$ and $n$

Figure 10: (3D-O3) with $m$ and $n$

( $n$ D-O3) Given two lines $m$ and $n$, fold $\mathcal{O}$ across a fold-line that superposes $m$ and $n$. There are two ways to generalize (O3) to $n$-dimensional case: leaving line parameters $m$ and $n$ as fold-lines, or treating them as $(n-1)$-dimensional hyperplanes. Let us first consider the case of two fold-lines (2D-hyperplane). We can make the two fold-lines coplanar included in (n-1)-fold-hyperplane as we treated in (3D-O3). Next we consider the case that $m$ and $n$ are hyper-half-planes. Roughly speaking, they will generally intersect in a fold-line. The hyperplane that bisects this dihedral angle is the desired fold-hyperplane.

(nD-O4) Given a point $P$ and a line $m$, fold $\mathcal{O}$ across a unique (n-1)-dimensional hyperplane orthogonal to $m$ that passes through point $P$.

This rule remains correct as stated for $n$-dimension case. There is a one parameter fam- 
ily of hyperplanes orthogonal to $m$. As the fold-hyperplane passes through $P$, the foldhyperplane is made unique.

( $n$ D-O5) Given a line $m$, a point $P$ not on $m$, and a point $Q$, where $m, P$ and $Q$ are on the same plane, fold $\mathcal{O}$ along a fold-plane passing through $Q$ to superpose $P$ and $m$.

This is the same as (3D-O5). If we supposed that $m, P$ and $Q$ are on the same (n-1)hyperplane, we could have $(n-1)$ parameter family of fold-(n-1)-hyperplane. It is not a hyperplane eligible for fold operation. The same consideration is applicable for the rest of the elementary fold operations. Thus we have the following.

( $n \mathrm{D}-\mathrm{O6})$ the same as (3D-O6)

( $n \mathrm{D}-\mathrm{O} 7)$ the same as (3D-O7)

\section{Concluding Remarks}

By extending Huzita-Justin fold operations, we presented a new fold principle for three- and higher-dimensional origami. The next step of our research is algebraic formulation of all the explained statements as is done in [8]. Then we will be able to precise the constraints in algebraic terms on the parameters of each statement.

Another interesting topics of research in this context is to explore more fold operations for higher-dimension origamis. Even with 3D origami, classical fold methods for recreational and artistic origami, such as inside (and outside) reverse fold and squash fold, can be given a new interpretation in our 3D origami. It would be natural to expect more fold operations for 3D and higher-dimensional origami.

As for the realization of the higher-dimensional origami, a work is in progress to incorporate the 3D origami based on this new principle in the computational origami system Eos. As a computation and proof engines, we introduced a layer of geometric algebra for systematic implementation[11]. Computer assisted construction of 3-dimensional origami examples using conformal geometric algebra is reported [15], where Huzita-Justin fold principle for 2D Origami is used.

\section{Acknowledgments}

This work was supported by JSPS KAKENHI Grants Numbers 25330007 and 16K00008 and by the Natural Sciences and Engineering Research Council of Canada.

\section{References}

[1] Hisashi Abe. Trisecting an Angle by Origami. Sugaku Seminar, page cover page, July 1980. http://www.phoenix-c.or.jp/ tokioka/n!/suu(underscore)semi.html.

[2] Roger C. Alperin. A Mathematical Theory of Origami Constructions and Numbers. New York Journal of Mathematics, 6:119-133, 2000.

[3] Roger. C. Alperin. Axioms for Origami and Compass Constructions. Journal for Geometry and Graphics, 20(1):13 - 22, January 2016.

[4] Margherita Piazzolla Beloch. Sul metodo del ripiegamento della carta per la risoluzione dei problemi geometrici. Periodico di Mathematiche, Serie IV, 16:104-108, 1936. 
[5] David. A. Cox. Galois Theory. Wiley-Interscience, 2004.

[6] Harold Scott MacDonald Coxeter. Regular Polytopes. Methuen, 1947.

[7] Fadoua Ghourabi, Tetsuo Ida, Hidekazu Takahashi, Mircea Marin, and Asem Kasem. Logical and algebraic view of huzita's origami axioms with applications to computational origami. In Proceedings of the 22nd ACM Symposium on Applied Computing, pages 767-772. ACM Press, 2007.

[8] Fadoua Ghourabi, Asem Kasem, and Cezary Kaliszyk. Algebraic Analysis of Huzita's Origami Operations and their Extensions. In Tetsuo Ida and Jacques Fleuriot, editors, Automated Deduction in Geometry, volume 7993 of LNAI, pages 143-160. Springer Heidelberg New York Dordrecht London, 2013.

[9] Humiaki Huzita. Axiomatic development of origami geometry. In Humiaki Huzita, editor, Proceedings of the First International Meeting of Origami Science and Technology, pages 143 - 158, Ferrara, Italy, December 1989.

[10] Humiaki Huzita. The trisection of a given angle solved by the geometry of origami. In Humiaki Huzita, editor, Proceedings of the First International Meeting of Origami Science and Technology, pages 195 - 214, Ferrara, Italy, December 1989.

[11] Tetsuo Ida, Jacques Fleuriot, and Fadoua Ghourabi. A new formalization of origami in geometric algebra. In Julien Narboux, Pascal Schreck, and Ileana Streinu, editors, ADG 2016. Eleventh International Workshop on Automated Deduction in Geometry, pages 117-136, June 2016.

[12] Tetsuo Ida and Hidekazu Takahashi. Origami fold as algebraic graph rewriting. J. Symb. Comput., 45(4):393-413, 2010.

[13] Tetsuo Ida, Dorin Tepeneu, Bruno Buchberger, and Judit Robu. Proving and Constraint Solving in Computational Origami. In Proceeding of the 7th International Symposium on Artificial Intelligence and Symbolic Computation (AISC 2004), volume 3249 of Lecture Notes in Artificial Intelligence, pages 132-142, 2004.

[14] Jaques Justin. Résolution par le pliage de l'équation du troisième degré et applications géométriques. In Humiaki Huzita, editor, Proceedings of the First International Meeting of Origami Science and Technology, pages 251-261, Ferrara, Italy, December 1989.

[15] Mitsuhiro Kondo, Takuya Matsuo, Yoshihiro Mizoguchi, and Hiroyuki Ochiai. A mathematica module for conformal geometric algebra and origami folding. In James H. Davenport and Fadoua Ghourabi, editors, SCSS 2016. 7th International Symposium on Symbolic Computation in Software Science, volume 39 of EPiC Series in Computing, pages 68-80. EasyChair, 2016.

[16] George E. Martin. Geometric Constructions. Springer-Verlag New York, Inc., 1998.

[17] Pierre L. Wantzel. Recherches sur les moyens de connaître si un problème de géométrie peut se résoudre avec la règle et le compas. Journal de Mathématiques Pures et Appliquées, pages 366-372, 1837. 